\title{
The Finite Element Approximation of a Coupled Reaction- Diffusion Problem with Non-Lipschitz Nonlinearities
}

\author{
John W. Barrett \& Patrick A. Wood, ${ }^{*}$ \\ Department of Mathematics, Imperial College, \\ London $S W 7$ 2BZ, England
}

October 4, 1995

\begin{abstract}
A coupled semilinear elliptic problem modelling an irreversible, isothermal chemical reaction is introduced, and discretised using the usual piecewise linear Galerkin finite element approximation. An interesting feature of the problem is that a reaction order of less than one gives rise to a "dead core" region. Initially, one reactant is assumed to be acting as a catalyst and is kept constant. It is shown that error bounds previously obtained for a scheme involving numerical integration can be improved upon by considering a quadratic regularisation of the nonlinear term.

This technique is then applied to the full coupled problem, and optimal $H^{1}$ and $L^{\infty}$ error bounds are proved in the absence of quadrature. For a scheme involving numerical integration, bounds similar to those obtained for the catalyst problem are shown to hold.
\end{abstract}

* Supported by a Research Studentship from the SERC 


\section{Introduction}

Consider two chemicals combining together in an irreversible, isothermal chemical reaction. By a mass-balancing argument, see [3] or [19] for example, it can be shown that this process may be modelled in terms of the concentration of the reactants as follows:

Given a bounded, connected, Lipschitz domain $\Omega \subset \mathbf{R}^{2}$, non-negative functions $a$ and $b$, constants $\lambda>0$ and $k>0$, find $u$ and $v$ such that

$$
-\Delta u+\lambda f(u) g(v)=0 \quad \text { in } \Omega,\left.\quad u\right|_{\partial \Omega}=\left.a\right|_{\partial \Omega}
$$

and

$$
-\Delta v+k \lambda f(u) g(v)=0 \quad \text { in } \Omega,\left.\quad v\right|_{\partial \Omega}=\left.b\right|_{\partial \Omega},
$$

where the functions $f$ and $g$ have the following properties:

$$
\begin{aligned}
& f, g \in C^{0}(\mathbf{R}) \cap C^{1}(0, \infty) \text { are monotonically increasing } \\
& \text { and satisfy } f(t)=g(t)=0 \quad \forall t \leq 0,
\end{aligned}
$$

and there exist constants $C, \gamma, \varepsilon_{0} \in \mathbf{R}^{+}$and $p, q \in(0,1]$ such that

and

$$
f(t), g(t) \leq C\left(1+t^{\gamma}\right) \quad \forall t \geq 0,
$$

$$
\left.\begin{array}{l}
\left|f\left(t_{1}\right)-f\left(t_{2}\right)\right| \leq C\left|t_{1}-t_{2}\right|^{p}, \\
\left|g\left(t_{1}\right)-g\left(t_{2}\right)\right| \leq C\left|t_{1}-t_{2}\right|^{q},
\end{array}\right\} \forall t_{1}, t_{2} \in\left[0, \varepsilon_{0}\right] .
$$

For example, given $p^{\prime} \in(0, \infty)$,

$$
f(t):=[t]_{+}^{p^{\prime}}, \quad \text { where } \quad[t]_{+}:= \begin{cases}t & \text { if } t>0, \\ 0 & \text { otherwise }\end{cases}
$$

satisfies (1.2), and (1.3) with $\gamma=p^{\prime}$ and $p=\min \left\{p^{\prime}, 1\right\}$. Note that if $f$ or $g$ is only Hölder continuous at the origin, i.e. if $p \in(0,1)$ or $q \in(0,1)$, then there is the possibility of a "dead core", that is, a region of $\Omega$ in which the concentration of one of the reactants is zero-see [8]. Here, the positive constant $k$ takes into account the fact that the two substances may have different diffusivities, and that they may not combine in a one-to-one ratio.

To begin with, a simplification of (1.1) will be considered, in which $a$ and $b$ are constants, and $k$ is zero. Hence $v$ is constant, and may be thought of as a catalyst:

Given positive constants $\lambda, p$ and $a$, find $u$ such that

$$
\begin{aligned}
-\Delta u+\lambda f(u) & =0 \quad \text { in } \Omega, \\
\left.u\right|_{\partial \Omega} & =a,
\end{aligned}
$$


where $f$ satisfies the conditions (1.2) and (1.3).

The error analysis of the finite element approximation of (1.4) is well-known if $p=1$. However, for $p \in(0,1)$, a non-standard approach is required due to the nonLipschitz nature of the nonlinear term. Nochetto, in [15], proves an optimal $L^{\infty}$ error bound for the standard continuous piecewise linear finite element approximation by using a linear regularisation of $f$. Barrett and Shanahan, in [6], prove that optimal $H^{1}$ and $L^{\infty}$ error bounds also hold if no regularisation is used.

In Section 2 it is shown that the error estimates for a non-regularised scheme involving numerical integration obtained in [6] can be simplified and improved for a scheme involving a quadratic regularisation of the nonlinear term by adapting the techniques of Barrett and Knabner in [5].

In Section 3, the techniques used for (1.4) are adapted to deal with the double nonlinearity of the coupled problem (1.1) with $p \in(0,1)$ and $q \in(0,1)$, and error bounds similar to those obtained for (1.4) are shown to hold.

\section{The Catalyst Problem}

Throughout the following, $W^{m, q}(\Omega)$ will be the Sobolev space on $\Omega$ with norm $\|\cdot\|_{m, q, \Omega}$ and semi-norm $|\cdot|_{m, q, \Omega}$. For $q=2, W^{m, 2}(\Omega)$ will be denoted by $H^{m}(\Omega)$, with the associated norm and semi-norm written as, respectively, $\|\cdot\|_{m, \Omega}$, and $|\cdot|_{m, \Omega}$. Define $H_{0}^{1}(\Omega)$ to be the set $\left\{\psi \in H^{1}(\Omega): \psi=0\right.$ on $\left.\partial \Omega\right\}$, and note the Poincaré inequality, $\|\psi\|_{1, \Omega} \leq C|\psi|_{1, \Omega} \forall \psi \in H_{0}^{1}(\Omega)$. Throughout, $C$ will be a generic constant independent of $h$, the mesh parameter, and $\varepsilon$, the regularisation parameter.

The boundary of $\Omega$ will satisfy one of the following smoothness conditions:

B1 $\partial \Omega$ is convex polygonal or $C^{1,1} ; \quad$ or $\quad \mathbf{B 2} \partial \Omega$ is $C^{1,1}$.

Introduce the inverse Laplacian, $\mathcal{G} \in \mathcal{L}\left(H^{-1}(\Omega), H_{0}^{1}(\Omega)\right)$ satisfying, given any $\zeta \in H^{-1}(\Omega)$,

$$
\int_{\Omega} \nabla \mathcal{G} \zeta \cdot \nabla \psi=\int_{\Omega} \zeta \psi \quad \forall \psi \in H_{0}^{1}(\Omega)
$$

In fact, for $\partial \Omega$ satisfying $\mathrm{B} 1, \mathcal{G} \in \mathcal{L}\left(L^{2}(\Omega), H_{0}^{1}(\Omega) \cap H^{2}(\Omega)\right)$ by elliptic regularity. In addition, if $\mathrm{B} 2$ holds, then the Calderón-Zygmund inequality applies, see [12]: for all $r \in(1, \infty)$ and $\zeta \in L^{r}(\Omega)$,

$$
|\mathcal{G} \zeta|_{2, r, \Omega} \leq C(r)\|\zeta\|_{0, r, \Omega}
$$

where

$$
C(r):= \begin{cases}C(r-1)^{-1} & \text { if } 1<r \leq 2, \\ C r & \text { if } 2 \leq r<\infty,\end{cases}
$$

from tracing the constants in the singular integrals involved, see e.g. [20, p.48]

The following well-known maximum principle will be required; see [12]: 
Strong Maximum Principle (SMP): If $\psi \in H^{2}(\Omega)$ satisfies

$$
-\Delta \psi \geq 0 \text { in } \Omega \text {, and } \psi \geq 0 \text { on } \partial \Omega ; \text { then } \psi \geq 0 \text { in } \bar{\Omega} .
$$

In addition, if $\psi(x)=0$ for some $x \in \Omega$, then $\psi \equiv 0$ in $\Omega$.

The problem (1.4) is now given in its weak formulation as follows:

$$
\mathcal{Q} \ldots\left\{\begin{array}{l}
\text { Given constants } \lambda \text { and } a \in \mathbf{R}^{+}, \text {find } u \in H_{a}^{1}(\Omega) \text { such that } \\
\int_{\Omega} \nabla u \cdot \nabla \psi+\lambda \int_{\Omega} f(u) \psi=0 \quad \forall \psi \in H_{0}^{1}(\Omega)
\end{array}\right.
$$

where $f$ satisfies (1.2) and (1.3), and $H_{a}^{1}(\Omega):=\left\{\psi \in H^{1}(\Omega): \psi=a\right.$ on $\left.\partial \Omega\right\}$.

The existence of a solution of $\mathcal{Q}$ follows from consideration of the associated minimisation problem. Furthermore the monotonicity of $f$ yields that the solution is unique. As $u \equiv a-\lambda \mathcal{G} f(u)$, it follows from (2.1), (1.2) and (1.3), that

$$
\|u\|_{2, r, \Omega} \leq C(r)\|f(u)\|_{0, r, \Omega} \leq C(r)\left[1+\|u\|_{0, r \gamma, \Omega}^{\gamma}\right] \leq C(r)
$$

for $r=2$ under boundary assumption B1, and for all $r \in(1, \infty)$ under B2. It then follows from the SMP that

$$
0 \leq u \leq a \quad \text { in } \Omega
$$

Let $\Omega^{h}$ be a polygonal approximation of $\Omega$ defined by $\bar{\Omega}^{h} \equiv \bigcup_{\tau \in T^{h}} \bar{\tau}$, where $T^{h}$ is a quasi-uniform triangulation and $h$ is the maximum diameter (i.e. side-length) over all triangles $\tau$ of $T^{h}$. Note that $\Omega^{h} \equiv \Omega$ if $\Omega$ is convex polygonal. Let $I$ be the set of nodes and $\left\{x_{i}\right\}_{i \in I}$ the coordinates of the vertices of this triangulation. It is assumed that for all $h, x_{i} \in \partial \Omega^{h} \Rightarrow x_{i} \in \partial \Omega$, so that $\operatorname{dist}\left(\partial \Omega, \partial \Omega^{h}\right) \leq C h^{2}$, and, for ease of exposition, that $\Omega^{h} \subseteq \Omega$. (Note that this last stipulation is made purely for the purposes of simplifying the following analysis; it is not essential.) The following function spaces are introduced:

$$
\begin{gathered}
S^{h}:=\left\{\chi \in C\left(\bar{\Omega}^{h}\right):\left.\chi\right|_{\tau} \text { is linear } \forall \tau \in T^{h}\right\}, \\
S_{0}^{h}:=\left\{\chi \in S^{h}:\left.\chi\right|_{\partial \Omega^{h}}=0\right\}, \text { and } S_{a}^{h}:=\left\{\chi \in S^{h}:\left.\chi\right|_{\partial \Omega^{h}}=a\right\} .
\end{gathered}
$$

The following inverse inequality and standard discrete Sobolev imbedding result will be required later: for any $r \in[1, \infty]$,

$$
|\chi|_{1, r, \Omega^{h}} \leq C h^{-1}|\chi|_{0, r, \Omega^{h}} \quad \forall \chi \in S^{h} ;
$$

and

$$
\|\chi\|_{0, \infty, \Omega^{h}} \leq C\left(\ln \frac{1}{h}\right)^{\frac{1}{2}}|\chi|_{1, \Omega^{h}} \quad \forall \chi \in S_{0}^{h}
$$


It will be convenient to extend the elements of $S_{0}^{h}$ and $S_{a}^{h}$ to the whole of $\bar{\Omega}$ by defining

$$
\left.\chi\right|_{\bar{\Omega} \backslash \bar{\Omega}^{h}}:=\left.\chi\right|_{\partial \Omega^{h}}
$$

Thus $\Omega^{h}$ as a domain of integration may be replaced by $\Omega$ for the purposes of the error analysis. The following results will be required-see, for example, Lemma 3.2 of [4]:

$$
\text { and } \left.\begin{array}{rl}
\|\psi\|_{0, \Omega \backslash \Omega^{h}} & \leq C\left(h^{2}|\psi|_{1, \Omega}+h|\psi|_{0, \partial \Omega}\right) \quad \forall \psi \in H^{1}(\Omega) \\
\|\psi\|_{1, \Omega \backslash \Omega^{h}} & \leq C h\|\psi\|_{2, \Omega} \quad \forall \psi \in H^{2}(\Omega) .
\end{array}\right\}
$$

Let $\pi_{h}: C(\bar{\Omega}) \rightarrow S^{h}$ denote the interpolation operator such that for any $\psi \in$ $C(\bar{\Omega}), \pi_{h} \psi \in S^{h}$ satisfies $\pi_{h} \psi\left(x_{i}\right)=\psi\left(x_{i}\right) \forall i \in I$. The standard interpolation result, see $[9$, p.123], states that for $m=0$ or $1, s \in[1, \infty]$ and $r \in(1, \infty]$, provided that $W^{2, r}(\tau) \subseteq W^{m, s}(\tau)$,

$$
\left|\psi-\pi_{h} \psi\right|_{m, s, \tau} \leq C h^{2\left(\frac{1}{s}-\frac{1}{r}+1\right)-m}|\psi|_{2, r, \tau},
$$

$\forall \psi \in W^{2, r}(\tau)$, and $\forall \tau \in T^{h}$. Note that as $\Omega \subset \mathbf{R}^{2}$, this result also applies for $r=1$, due to the imbedding $W^{2,1}(\Omega) \hookrightarrow C(\bar{\Omega})$; see $[13$, p.300].

The approximate problem can now be stated as follows:

$$
\mathcal{Q}^{h} \ldots\left\{\begin{array}{l}
\text { Given the data of } \mathcal{Q}, \text { find } u^{h} \in S_{a}^{h} \text { such that } \\
\int_{\Omega^{h}} \nabla u^{h} \cdot \nabla \chi+\lambda \int_{\Omega^{h}} f\left(u^{h}\right) \chi=0 \quad \forall \chi \in S_{0}^{h} .
\end{array}\right.
$$

The existence of a solution of $\mathcal{Q}^{h}$ follows from consideration of the associated discrete minimisation problem. This solution is unique by the monotonicity of $f$.

If $p=1, f$ is a locally Lipschitz function. It is convenient to replace $f$ in $\mathcal{Q}^{h}$ by a truncation $\bar{f}$ defined as follows:

$$
\bar{f}(t):=\left\{\begin{array}{l}
f(t), \quad t \leq a, \\
f(a), \quad t>a .
\end{array}\right.
$$

The error analysis is then straightforward as $\bar{f}$ is globally Lipschitz-for details see [18] and [10].

Some further notation and corresponding results are now given. Introduce the elliptic projection operator $Q^{h} \in \mathcal{L}\left(H^{2}(\Omega), S^{h}\right)$ for general boundary data as follows: given $\psi \in H^{2}(\Omega)$, define $Q^{h} \psi \in S^{h}$ satisfying

$$
\left.\begin{array}{rl}
\left.Q^{h} \psi\right|_{\partial \Omega^{h}} & =\left.\pi_{h} \psi\right|_{\partial \Omega^{h}} \\
\left.Q^{h} \psi\right) \cdot \nabla \chi & =0 \quad \forall \chi \in S_{0}^{h} .
\end{array}\right\}
$$


The following error bound is standard: for $m=0$ or 1 and any $\psi \in H^{2}(\Omega)$,

$$
\left\|\left(I-Q^{h}\right) \psi\right\|_{m, \Omega^{h}} \leq C h^{2-m}|\psi|_{2, \Omega} .
$$

Also there exists $h_{0}$ such that for $\psi \in H_{0}^{1}(\Omega) \cap H^{2}(\Omega)$, for all $h<h_{0}$,

$$
\left\|\left(I-Q^{h}\right) \psi\right\|_{0, \infty, \Omega^{h}} \leq C\left(\ln \frac{1}{h}\right)\left\|\left(I-\pi_{h}\right) \psi\right\|_{0, \infty, \Omega^{h}} ;
$$

- see [17]. Combining (2.13), (2.9) and (2.1), with $r:=\ln 1 / h$, and noting (2.2) yields that if B2 holds, then for $h<h_{0}$ and for all $\zeta \in L^{\infty}(\Omega)$,

$$
\left\|\left(I-Q^{h}\right) \mathcal{G} \zeta\right\|_{0, \infty, \Omega} \leq C\left(h \ln \frac{1}{h}\right)^{2}\|\zeta\|_{0, \infty, \Omega}
$$

If $f$ is not locally Lipschitz, i.e. if $p \in(0,1)$, the standard error analysis yields a pessimistic result in the $H^{1}$ case, and is not applicable in the $L^{2}$ and $L^{\infty}$ cases. There is now the possibility of the formation of a dead core, that is, a subregion $\Omega_{0}$ of $\Omega$ in which $u \equiv 0$. $\mathcal{Q}$ may now be thought of as a free-boundary problem, since the location of $\partial \Omega_{0}$ is a priori unknown.

One way of dealing with the non-differentiability of $f$ at the origin is to "smooth out" the problem by replacing $f$ by a regularised function $f_{\varepsilon}$, as in [15]. The problem to be solved therefore becomes

$$
\mathcal{Q}_{\varepsilon} \ldots\left\{\begin{array}{l}
\text { Given } p \in(0,1) \text { and } a \in \mathbf{R}^{+}, \text {find } u_{\varepsilon} \in H_{a}^{1}(\Omega) \text { such that } \\
-\Delta u_{\varepsilon}+\lambda f_{\varepsilon}\left(u_{\varepsilon}\right)=0 \quad \text { in } \Omega
\end{array}\right.
$$

with corresponding discretisation

$$
\mathcal{Q}_{\varepsilon}^{h} \cdots\left\{\begin{array}{l}
\text { With data as for } \mathcal{Q}_{\varepsilon}, \text { find } u_{\varepsilon}^{h} \in S_{a}^{h} \text { such that } \\
\int_{\Omega^{h}} \nabla u_{\varepsilon}^{h} \cdot \nabla \chi+\lambda \int_{\Omega^{h}} f_{\varepsilon}\left(u_{\varepsilon}^{h}\right) \chi=0 \quad \forall \chi \in S_{0}^{h} .
\end{array}\right.
$$

In [15], Nochetto shows that for a linear regularisation $f_{\varepsilon}, u_{\varepsilon}$, the unique solution of $\mathcal{Q}_{\varepsilon}$, satisfies

$$
\left\|u-u_{\varepsilon}\right\|_{0, \infty, \Omega} \leq \varepsilon^{\frac{1}{1-p}},
$$

and that for $h<h_{0}$ and for any $\varepsilon>0$ the unique solution $u_{\varepsilon}^{h}$ of $\mathcal{Q}_{\varepsilon}^{h}$, satisfies

$$
\left\|u_{\varepsilon}-u_{\varepsilon}^{h}\right\|_{0, \infty, \Omega} \leq C\left(h \ln \frac{1}{h}\right)^{2}
$$

uniformly in $\varepsilon$. Thus, for $\varepsilon:=C(h \ln 1 / h)^{2(1-p)}$,

$$
\left\|u-u_{\varepsilon}^{h}\right\|_{0, \infty, \Omega} \leq C_{*}\left(h \ln \frac{1}{h}\right)^{2}
$$


Nochetto also obtains convergence estimates for the discrete free boundary under the assumption that the solution to the continuous problem satisfies the following non-degeneracy conditions:

Non-Degeneracy Conditions: There exist constants $\delta_{0}, C, M>0$ such that for $\delta \in\left(0, \delta_{0}\right)$,

$$
\text { NDC1: } \underline{m}\left(\Omega_{\delta}\right) \leq C \delta^{\frac{1}{2}},
$$

and

$$
\text { NDC2: } \quad \Omega_{\delta} \subseteq\left\{x \in \Omega \backslash \Omega_{0}: \operatorname{dist}\left(x, \partial \Omega_{0}\right) \leq M \delta^{\frac{1}{2}}\right\},
$$

where $\Omega_{\delta}:=\left\{x \in \Omega: 0<u(x) \leq \delta^{\frac{1}{1-p}}\right\}$, and $\Omega_{0}:=\{x \in \Omega: u(x)=0\}$.

For the model case $f(t)=[t]_{+}^{p}$, it follows from [16] that B1 is sufficient for NDC1 to hold, and from [2] and [11] that B2 plus convexity are sufficient conditions on $\Omega$ for NDC2 to hold.

In [14] and [15], Nochetto defines the discrete dead core as follows:

$$
\Omega_{0}^{h}:=\left\{x \in \Omega^{h}: u_{\varepsilon}^{h}(x) \leq C_{*}(h \ln 1 / h)^{2}\right\} .
$$

By (2.17), $\Omega_{0} \subseteq \Omega_{0}^{h}$. Under NDC1, Nochetto then proves that

$$
\underline{m}\left(\Omega_{0}^{h} \backslash \Omega_{0}\right) \leq C\left(h \ln \frac{1}{h}\right)^{1-p}
$$

and under NDC2 that

$$
\partial \Omega_{0}^{h} \subseteq\left\{x \in{\overline{\Omega \backslash \Omega_{0}}}_{0} \operatorname{dist}\left(x, \partial \Omega_{0}\right) \leq C(h \ln 1 / h)^{1-p}\right\} .
$$

In [6], Barrett and Shanahan show that optimal $L^{\infty}$ and $H^{1}$ error bounds, and hence analogous results to (2.18) and (2.19), also apply to $\mathcal{Q}^{h}$, the direct approximation of $\mathcal{Q}$, without regularisation. However, $\mathcal{Q}^{h}$, like $\mathcal{Q}_{\varepsilon}^{h}$, involves exact integration of the nonlinear term, and as it is virtually impossible to implement such a scheme in practice, it is of interest to introduce and analyse a method involving numerical integration. Error bounds are proved in [6] for such a scheme without regularisation, but it is now shown that these can be improved upon by using a quadratic regularisation of $f$ and applying the techniques of Barrett and Knabner in [5].

A quadrature rule is introduced as follows: given a triangle $\tau \in T^{h}$ having vertices $\left\{a_{i}\right\}_{i=1}^{3}$, for $\psi \in C(\bar{\tau})$, define

$$
Q_{\tau}(\psi):=\frac{1}{3} \underline{m}(\tau) \sum_{i=1}^{3} \psi\left(a_{i}\right) .
$$

Then

$$
\int_{\Omega^{h}} \psi_{1} \psi_{2} \approx\left(\psi_{1}, \psi_{2}\right)^{h}:=\sum_{\tau \in T^{h}} Q_{\tau}\left(\psi_{1} \psi_{2}\right)
$$


for all $\psi_{1}, \psi_{2} \in C\left(\bar{\Omega}^{h}\right)$. Note the following result: for $\chi_{1}, \chi_{2} \in S^{h}$,

$$
\left|\int_{\tau} \chi_{1} \chi_{2}-Q_{\tau}\left(\chi_{1} \chi_{2}\right)\right| \leq C h^{2}\left|\chi_{1}\right|_{1, \tau}\left|\chi_{2}\right|_{1, \tau}
$$

for all $\tau \in T^{h}$. It now follows from (2.21), (2.9) and (2.6) that for all $\psi \in W^{2,1}(\Omega)$ and $\chi \in S_{0}^{h}$,

$$
\begin{aligned}
\left|\int_{\Omega^{h}} \psi \chi-(\psi, \chi)^{h}\right| & =\left|\int_{\Omega^{h}}\left[\left(I-\pi_{h}\right) \psi\right] \chi+\int_{\Omega^{h}}\left(\pi_{h} \psi\right) \chi-\left(\pi_{h} \psi, \chi\right)^{h}\right| \\
& \leq\left.\left|\left(I-\pi_{h}\right) \psi\right|_{0,1, \Omega^{h}}|| \chi\right|_{0, \infty, \Omega^{h}}+C h^{2}\left|\pi_{h} \psi\right|_{1, \Omega^{h}}|\chi|_{1, \Omega^{h}} \\
& \leq C h^{2}\left[\left(\ln \frac{1}{h}\right)^{\frac{1}{2}}|\psi|_{2,1, \Omega^{h}}+\left|\pi_{h} \psi\right|_{1, \Omega^{h}}\right]|\chi|_{1, \Omega^{h}} .
\end{aligned}
$$

Also required is the fact that for all $\chi \in S^{h}$,

$$
\|\chi\|_{0, \Omega^{h}}^{2} \leq(\chi, \chi)^{h} \leq C\|\chi\|_{0, \Omega^{h}}^{2}
$$

The standard Galerkin finite element approximation of $\mathcal{Q}$ in the presence of numerical integration is now defined as follows:

$$
\hat{\mathcal{Q}}^{h} \ldots\left\{\begin{array}{l}
\text { Given } p \in(0,1) \text { and } a \in \mathbf{R}^{+}, \text {find } \hat{u}^{h} \in S_{a}^{h} \text { such that } \\
\int_{\Omega^{h}} \nabla \hat{u}^{h} \cdot \nabla \chi+\lambda\left(f\left(\hat{u}^{h}\right), \chi\right)^{h}=0 \quad \forall \chi \in S_{0}^{h} .
\end{array}\right.
$$

It is proved in [6] that in two dimensions, $u$ and $\hat{u}^{h}$, the unique solutions of, respectively, $\mathcal{Q}$ and $\hat{\mathcal{Q}}^{h}$, satisfy the following error estimates: provided that $u$, the solution of the continuous problem, satisfies NDC2, then for $h<h_{0}$,

$$
\left|u-\hat{u}^{h}\right|_{1, \Omega^{h}} \leq C h
$$

and

$$
\left\|u-\hat{u}^{h}\right\|_{0, \infty, \Omega^{h}} \leq\left\{\begin{array}{ll}
C h^{\frac{2}{2-p}} \ln \frac{1}{h}, & \frac{1}{3} \leq p<1, \\
C h^{\frac{1+p}{(1-p)(2-p)}} \ln \frac{1}{h}, & 2-\sqrt{3} \leq p \leq \frac{1}{3} \\
C h \ln \frac{1}{h}, & 0<p \leq 2-\sqrt{3} .
\end{array} .\right.
$$

The following analysis improves the bound (2.25) in the case $p<\frac{1}{3}$, and moreover, does not require the assumption of non-degeneracy.

In order to define a suitable regularisation of the nonlinear term, additional restrictions will now be required on the function $f$. Further to (1.2) and (1.3), it is assumed that

$$
f \in C^{2}(0, \infty) \text { and } f^{\prime \prime}(t) \leq 0 \forall t>0
$$


Note that this assumption holds for the model case $f(t) \equiv[t]_{+}^{p}$.

The regularisation $f_{\varepsilon}$ of $f$ will need to satisfy the following conditions: Given $\varepsilon \in\left(0, \varepsilon_{0}\right)$,

$$
\begin{aligned}
& \text { (i) } f_{\varepsilon}(t) \equiv f(t) \text { for } t \notin\left(0, \varepsilon^{\frac{1}{1-p}}\right) \text {; } \\
& \text { (ii) } f_{\varepsilon}(t) \text { is strictly monotonically increasing on }\left(0, \varepsilon^{\frac{1}{1-p}}\right) \text {; } \\
& \text { (iii) } f_{\varepsilon}(t) \in C^{1,1}[0, \infty) \text {, and } f_{\varepsilon}^{\prime}(t) \leq C \varepsilon^{-1} \forall t \geq 0 ; \\
& \text { (iv) } f_{\varepsilon}^{\prime \prime}(t) \leq 0 \text { for almost all } t \geq 0 .
\end{aligned}
$$

These conditions are satisfied if $f_{\varepsilon}$ is chosen to be quadratic on $\left[0, \varepsilon^{\frac{1}{1-p}}\right]$, as follows:

$$
f_{\varepsilon}(t):= \begin{cases}\alpha_{0} t^{2}+\beta_{0} t & \text { if } 0 \leq t \leq \varepsilon^{\frac{1}{1-p}}, \\ f(t) & \text { otherwise }\end{cases}
$$

where $\alpha_{0}:=\varepsilon^{-\frac{1}{1-p}} f^{\prime}\left(\varepsilon^{\frac{1}{1-p}}\right)-\varepsilon^{-\frac{2}{1-p}} f\left(\varepsilon^{\frac{1}{1-p}}\right)$, and $\beta_{0}:=2 \varepsilon^{-\frac{1}{1-p}} f\left(\varepsilon^{\frac{1}{1-p}}\right)-f^{\prime}\left(\varepsilon^{\frac{1}{1-p}}\right)$ are chosen so that $f_{\varepsilon}(t)$ and $f_{\varepsilon}^{\prime}(t)$ are continuous for $t \geq 0$. For example, if $f$ is the power function $[t]_{+}^{p}$, then $\alpha_{0}=(p-1) \varepsilon^{\frac{p-2}{1-p}}$, and $\beta_{0}=(2-p) \varepsilon^{-1}$.

The bounds (2.15) and (2.16) obtained by Nochetto for a linear regularisation depend on the length of the interval $I \subset[0, \infty)$ over which $f$ and $f_{\varepsilon}$ differ, and they apply unchanged for the present quadratic regularisation.

The existence and uniqueness of a solution to $\mathcal{Q}_{\varepsilon}$ follow as for the non-regularised problem. Similarly to $(2.3)$, it follows from (2.1), the definition of $f_{\varepsilon}$ and (1.3), that

$$
\left\|u_{\varepsilon}\right\|_{2, r, \Omega} \leq C(r)\left\|f_{\varepsilon}\left(u_{\varepsilon}\right)\right\|_{0, r, \Omega} \leq C(r),
$$

for $r=2$ under boundary assumption B1, and for all $r \in(1, \infty)$ under B2. Hence it is an immediate consequence of the SMP that

$$
0 \leq u_{\varepsilon} \leq a \quad \text { on } \Omega
$$

The discrete, regularised problem in the presence of numerical integration is as follows:

$$
\hat{\mathcal{Q}}_{\varepsilon}^{h} \cdots\left\{\begin{array}{l}
\text { Given } p \in(0,1) \text { and } a \in \mathbf{R}^{+}, \text {find } \hat{u}_{\varepsilon}^{h} \in S_{a}^{h} \text { such that } \\
\int_{\Omega^{h}} \nabla \hat{u}_{\varepsilon}^{h} \cdot \nabla \chi+\lambda\left(f_{\varepsilon}\left(\hat{u}_{\varepsilon}^{h}\right), \chi\right)^{h}=0 \quad \forall \chi \in S_{0}^{h} .
\end{array}\right.
$$

The existence of a solution of $\hat{\mathcal{Q}}_{\varepsilon}^{h}$ follows by considering the appropriate minimisation problem. This solution is unique by the monotonicity of $f_{\varepsilon}$. 
Lemma 2.1 Under boundary assumption B1,

$$
\left|\hat{u}_{\varepsilon}^{h}-Q^{h} u_{\varepsilon}\right|_{1, \Omega} \leq C \varepsilon^{-1} h^{2}\left(\ln \frac{1}{h}\right)^{\frac{1}{2}} .
$$

Proof: Define $E:=\hat{u}_{\varepsilon}^{h}-Q^{h} u_{\varepsilon} \in S_{0}^{h}$. From (2.11), $\hat{\mathcal{Q}}_{\varepsilon}^{h}$ and $\mathcal{Q}_{\varepsilon}^{h}$, the monotonicity of $f_{\varepsilon},(2.22)$, the Mean Value Theorem, (2.27) (iii), (2.23), discrete Sobolev imbedding (2.6), Poincaré's inequality, (2.12) and (2.9),

$$
\begin{aligned}
|E|_{1, \Omega}^{2} & =\lambda \int_{\Omega^{h}} f_{\varepsilon}\left(u_{\varepsilon}\right) E-\lambda\left(f_{\varepsilon}\left(u_{\varepsilon}\right), E\right)^{h}+\lambda\left(f_{\varepsilon}\left(u_{\varepsilon}\right)-f_{\varepsilon}\left(\hat{u}_{\varepsilon}^{h}\right), \hat{u}_{\varepsilon}^{h}-Q^{h} u_{\varepsilon}\right)^{h} \\
& \leq \lambda \int_{\Omega^{h}} f_{\varepsilon}\left(u_{\varepsilon}\right) E-\lambda\left(f_{\varepsilon}\left(u_{\varepsilon}\right), E\right)^{h}+\lambda\left(f_{\varepsilon}\left(u_{\varepsilon}\right)-f_{\varepsilon}\left(\hat{u}_{\varepsilon}^{h}\right),\left(I-Q^{h}\right) u_{\varepsilon}\right)^{h} \\
& \leq C h^{2}\left[\left(\ln \frac{1}{h}\right)^{\frac{1}{2}}\left|f_{\varepsilon}\left(u_{\varepsilon}\right)\right|_{2,1, \Omega^{h}}+\left|\pi_{h} f_{\varepsilon}\left(u_{\varepsilon}\right)\right|_{1, \Omega^{h}}\right]|E|_{1, \Omega^{h}}+ \\
C \varepsilon^{-1}||\left(\pi_{h}-Q^{h}\right) u_{\varepsilon} \|_{0, \Omega^{h}}\left[\left\|\left(\pi_{h}-Q^{h}\right) u_{\varepsilon}\right\|_{0, \Omega^{h}}+|E|_{1, \Omega^{h}}\right] & \leq C h^{2}\left(\ln \frac{1}{h}\right)^{\frac{1}{2}}|E|_{1, \Omega^{h}}\left(\left|f_{\varepsilon}\left(u_{\varepsilon}\right)\right|_{2,1, \Omega^{h}}+\varepsilon^{-1}\right)+C \varepsilon^{-1} h^{4},
\end{aligned}
$$

where (2.9), Sobolev imbedding, (2.27) (iii) and (2.29) have been used to note that

$$
\left|\pi_{h} f_{\varepsilon}\left(u_{\varepsilon}\right)\right|_{1, \Omega^{h}} \leq C \|\left. f_{\varepsilon}\left(u_{\varepsilon}\right)\right|_{2,1, \Omega^{h}} \leq C\left(\left|f_{\varepsilon}\left(u_{\varepsilon}\right)\right|_{2,1, \Omega^{h}}+\varepsilon^{-1}\right) .
$$

Now, by expansion (see Theorem 7.8 and $\S 7.5$ in [12]) and (2.27)(iii),

$$
\left|f_{\varepsilon}\left(u_{\varepsilon}\right)\right|_{2,1, \Omega^{h}} \leq C\left(\left|f_{\varepsilon}^{\prime \prime}\left(u_{\varepsilon}\right)\left(\nabla u_{\varepsilon}\right)^{2}\right|_{0,1, \Omega}+\varepsilon^{-1}\left|u_{\varepsilon}\right|_{2,1, \Omega}\right)
$$

and since $f_{\varepsilon}^{\prime \prime}(t) \leq 0$ for almost all $t \geq 0$, it follows from (2.27)(iii), a trace inequality and (2.29) that

$$
\begin{aligned}
\left|f_{\varepsilon}^{\prime \prime}\left(u_{\varepsilon}\right)\left(\nabla u_{\varepsilon}\right)^{2}\right|_{0,1, \Omega} & =-\int_{\Omega} \nabla f_{\varepsilon}^{\prime}\left(u_{\varepsilon}\right) \cdot \nabla u_{\varepsilon} \\
& =\int_{\Omega} f_{\varepsilon}^{\prime}\left(u_{\varepsilon}\right) \Delta u_{\varepsilon}-\int_{\partial \Omega} f_{\varepsilon}^{\prime}\left(u_{\varepsilon}\right) \nabla u_{\varepsilon} \cdot \underline{\mathbf{n}} \\
& \leq C \varepsilon^{-1}\left\|u_{\varepsilon}\right\|_{2,1, \Omega} \leq C \varepsilon^{-1},
\end{aligned}
$$

where $\underline{\mathbf{n}}$ is defined to be the outward unit normal to $\partial \Omega$. Combining (2.31), (2.32) and (2.33), yields the desired result.

Theorem 2.1 Under boundary assumption BD, for $\varepsilon=C\left(h^{2} \ln 1 / h\right)^{\frac{1-p}{2-p}}$, there exist $\hat{C}_{*}, h_{0}>0$ such that for all $h<h_{0}$,

$$
\left\|u-\hat{u}_{\varepsilon}^{h}\right\|_{0, \infty, \Omega} \leq \hat{C}_{*}\left(h^{2} \ln \frac{1}{h}\right)^{\frac{1}{2-p}} .
$$


Thus, for the discrete dead core, $\hat{\Omega}_{0}^{h}:=\left\{x \in \Omega^{h}: \hat{u}_{\varepsilon}^{h}(x) \leq \hat{C}_{*}\left(h^{2} \ln 1 / h\right)^{\frac{1}{2-p}}\right\}$, it follows that under the assumption NDC1, $\underline{m}\left(\hat{\Omega}_{0}^{h} \backslash \Omega_{0}\right) \leq C\left(h^{2} \ln 1 / h\right)^{\frac{1-p}{2(2-p)}}$, and that under NDCD, $\partial \hat{\Omega}_{0}^{h} \subseteq\left\{x \in \overline{\Omega \backslash \Omega}_{0}\right.$ : $\left.\operatorname{dist}\left(x, \partial \Omega_{0}\right) \leq C\left(h^{2} \ln 1 / h\right)^{\frac{1-p}{2(2-p)}}\right\}$.

Proof: By discrete Sobolev imbedding (2.6), Lemma 2.1, (2.14) and (2.30), noting that $u_{\varepsilon}=a-\lambda \mathcal{G} f_{\varepsilon}\left(u_{\varepsilon}\right)$,

$$
\left\|u_{\varepsilon}-\hat{u}_{\varepsilon}^{h}\right\|_{0, \infty, \Omega} \leq C\left(\varepsilon^{-1}+\ln \frac{1}{h}\right) h^{2} \ln \frac{1}{h} \leq C \varepsilon^{-1} h^{2} \ln \frac{1}{h} .
$$

Combining (2.15) and (2.35), and setting $\varepsilon^{\frac{1}{1-p}}=C\left(h^{2} \ln 1 / h\right)^{\frac{1}{2-p}}$, yields the desired result (2.34).

Convergence of the discrete free boundary now follows immediately from (2.34) and non-degeneracy.

Note that the $L^{\infty}$ bound of Theorem 2.1 does not require a non-degeneracy condition, and that for $p<\frac{1}{3}$, it is an improvement on (2.25).

An $H^{1}$ bound requires the following result:

$$
\left|u-u_{\varepsilon}\right|_{1, \Omega} \leq C\left[\underline{m}\left(\Omega_{\varepsilon}\right)\right]^{\frac{1}{2}} \varepsilon^{\frac{1+p}{2(1-p)}},
$$

where $\Omega_{\varepsilon}:=\left\{x \in \Omega: 0<u(x) \leq \varepsilon^{\frac{1}{1-p}}\right\}$. This is easily deduced from $\mathcal{Q}, \mathcal{Q}_{\varepsilon}$ and the monotonicity of $f_{\varepsilon}$. Combining (2.36), (2.12), (2.8), (2.29) and Lemma 2.1 yields the following result:

Lemma 2.2 Under assumption $B 1$, for $\varepsilon=C\left(h^{2} \ln 1 / h\right)^{\frac{1-p}{2-p}}$,

$$
\left|u-\hat{u}_{\varepsilon}^{h}\right|_{1, \Omega} \leq \begin{cases}C h, & p>\frac{1}{2}, \\ C\left(h^{2} \ln \frac{1}{h}\right)^{\frac{1+p}{2(2-p)}} \quad, \quad 0<p \leq \frac{1}{2} .\end{cases}
$$

Moreover, if NDC1 holds, then

$$
\left|u-\hat{u}_{\varepsilon}^{h}\right|_{1, \Omega} \leq\left\{\begin{array}{ll}
C h, & p>\frac{1}{3}, \\
C\left(h^{2} \ln \frac{1}{h}\right)^{\frac{3+p}{4(2-p)}}, & 0<p \leq \frac{1}{3} .
\end{array} \bullet\right.
$$

Note that under the assumption of NDC2, it is proved in [6] that (2.24) holds, and it follows exactly as for (2.36) that

$$
\left|\hat{u}^{h}-\hat{u}_{\varepsilon}^{h}\right|_{1, \Omega} \leq C \varepsilon^{\frac{1+p}{2(1-p)}} .
$$

So by choosing $\varepsilon:=C h^{\frac{2(1-p)}{1+p}}$ and noting (2.8), it follows that

$$
\left|u-\hat{u}_{\varepsilon}^{h}\right|_{1, \Omega} \leq C h .
$$

Note that according to the above analysis, the choice of $\varepsilon$ is determined by whether greater accuracy is required in $L^{\infty}$ or $H^{1}$. 


\section{The Coupled Problem}

Consider now the coupled problem (1.1), with $a$ and $b$ in $W^{2, \infty}(\Omega)$, and with the additional requirement that $f(g)$ satisfies $(2.26)$, if $p(q)<1$. This problem will be referred to henceforth as $\mathcal{Q}$.

The technique used in [7] and [8] is to write $w:=k u-v$. From $\mathcal{Q}, w$ satifies

$$
\left\{\begin{aligned}
\Delta w & =0 \quad \text { in } \Omega, \\
\left.w\right|_{\partial \Omega} & =c:=\left.(k a-b)\right|_{\partial \Omega},
\end{aligned}\right.
$$

and so exists uniquely. The system $\mathcal{Q}$ then becomes

$$
\left\{\begin{array}{rlrl}
-\Delta u+\lambda f(u) g(k u-w) & =0 & \text { in } \Omega, & \left.u\right|_{\partial \Omega}=\left.a\right|_{\partial \Omega} \\
-\Delta v+k \lambda f((v+w) / k) g(v) & =0 & \text { in } \Omega, & \left.v\right|_{\partial \Omega}=\left.b\right|_{\partial \Omega}
\end{array} .\right.
$$

By considering the associated minimisation problems, it is not difficult to show that the solution of (3.2), and hence $\mathcal{Q}$, exists. Uniqueness follows from the monotonicity of $f$ and $g$. Note that $u \equiv a+\mathcal{G}[\Delta a-\lambda f(u) g(v)]$, and $v \equiv b+\mathcal{G}[\Delta b-k \lambda f(u) g(v)]$. Then similarly to (2.3), it follows from $(2.1),(1.2)$ and (1.3), that

$$
\left.\begin{array}{l}
\|u\|_{2, r, \Omega} \\
\|v\|_{2, r, \Omega}
\end{array}\right\} \leq C(r)\left[1+\|u\|_{0,2 r \gamma, \Omega}^{\gamma}\right]\left[1+\|v\|_{0,2 r \gamma, \Omega}^{\gamma}\right] \leq C(r)
$$

for $r=2$ under boundary assumption B1, and for all $r \in(1, \infty)$ under B2. It thus follows immediately from the SMP that

$$
\left.\begin{array}{ll}
0 \leq u \leq\|a\|_{0, \infty, \partial \Omega} \\
\text { and } \quad \\
0 \leq v \leq\|b\|_{0, \infty, \partial \Omega}
\end{array}\right\} \text { on } \Omega
$$

There are two possibilities: either $c$ does not change sign on $\partial \Omega$, in which case it follows from (3.4) and the SMP applied to $w$ that there can be at most one dead core, or $c$ does change sign on $\partial \Omega$, and two dead cores may exist. It is this second case which is of most interest, although the following analysis copes with both.

It will be assumed for convenience and without loss of generality that $p$ and $q$ in the definitions of $f$ and $g$ satisfy $p \leq q$. There are thus three possibilities:

$$
\text { (i) } p=q=1 \text {; } \quad \text { (ii) } 0<p<q=1 \text {; } \quad \text { (iii) } 0<p \leq q<1 \text {. }
$$

Clearly case (i) requires no regularisation, and in case (ii) only $f$ need be regularised. The following analysis is formulated specifically to deal with case (iii), but is readily adapted to deal with cases (i) and (ii). 
The non-Lipschitz functions $f$ and $g$ are replaced by, respectively, quadratic regularisations identical to the one introduced in Section 2. Note that

$$
\left.\begin{array}{l}
\sup _{t \geq 0}\left|f_{\varepsilon}^{\prime}(t)\right| \\
\sup _{t \geq 0}\left|g_{\varepsilon}^{\prime}(t)\right|
\end{array}\right\} \leq C \varepsilon^{-1} .
$$

The regularised problem is now:

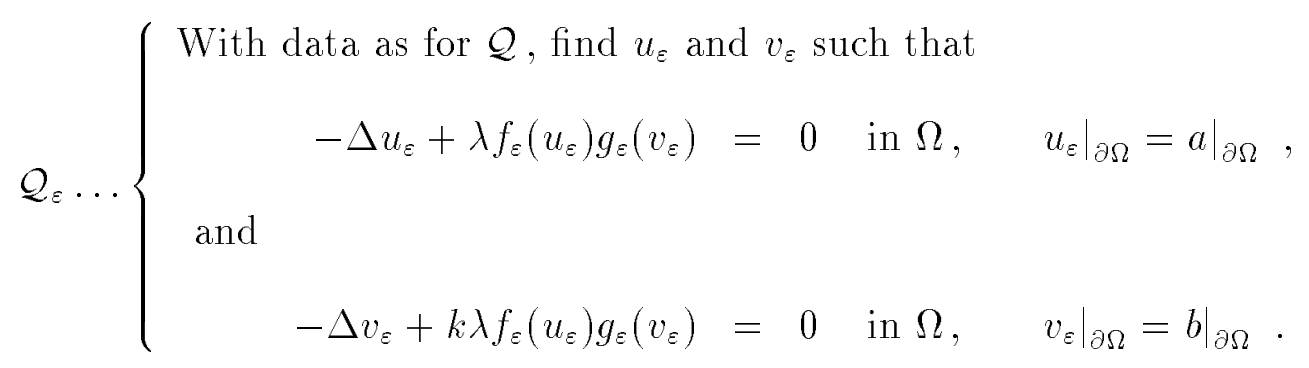

Note that $w_{\varepsilon}:=k u_{\varepsilon}-v_{\varepsilon} \equiv w$ by uniqueness for Laplace's equation. Existence and uniqueness of a solution of $\mathcal{Q}_{\varepsilon}$ follow exactly as for $\mathcal{Q}$, and by (2.1), the definitions of $f_{\varepsilon}$ and $g_{\varepsilon}$, and (1.3),

$$
\left.\begin{array}{l}
\left\|u_{\varepsilon}\right\|_{2, r, \Omega} \\
\left\|v_{\varepsilon}\right\|_{2, r, \Omega}
\end{array}\right\} \leq C(r)\left[1+\left\|u_{\varepsilon}\right\|_{0,2 r \gamma, \Omega}^{\gamma}\right]\left[1+\left\|v_{\varepsilon}\right\|_{0,2 r \gamma, \Omega}^{\gamma}\right] \leq C(r),
$$

for $r=2$ under boundary assumption B1, and for all $r \in(1, \infty)$ under B2. Hence, from the SMP,

$$
\text { and } \left.\begin{array}{l}
0 \leq u_{\varepsilon} \leq\|a\|_{0, \infty, \partial \Omega} \\
0 \leq v_{\varepsilon} \leq\|b\|_{0, \infty, \partial \Omega}
\end{array}\right\} \text { on } \Omega
$$

The following result adapts the method used by Nochetto in [15].

\section{Lemma 3.1}

$$
\left\|u-u_{\varepsilon}\right\|_{0, \infty, \Omega}+\left\|v-v_{\varepsilon}\right\|_{0, \infty, \Omega} \leq C \varepsilon^{\frac{1}{1-p}},
$$

recalling that for definiteness, $p \leq q$.

Proof: Let $e_{\varepsilon}:=u-u_{\varepsilon}$. From $\mathcal{Q}, \mathcal{Q}_{\varepsilon}$ and the definition of $w$, for all $\psi \in H_{0}^{1}(\Omega)$,

$$
\int_{\Omega} \nabla e_{\varepsilon} \cdot \nabla \psi=\lambda \int_{\Omega}\left[f_{\varepsilon}\left(u_{\varepsilon}\right) g_{\varepsilon}\left(k u_{\varepsilon}-w\right)-f(u) g(k u-w)\right] \psi
$$

From Poincaré's inequality and (3.8), it follows that for any positive integer $n$,

$$
\begin{aligned}
& \left\|e_{\varepsilon}\right\|_{0,2(n+1), \Omega}^{2(n+1)} \leq C\left\|e_{\varepsilon}^{n+1}\right\|_{1, \Omega}^{2}=C \frac{(n+1)^{2}}{2 n+1} \int_{\Omega} \nabla e_{\varepsilon} \cdot \nabla\left(e_{\varepsilon}^{2 n+1}\right) \\
= & C \frac{(n+1)^{2}}{2 n+1} \lambda \int_{\Omega}\left[f_{\varepsilon}\left(u_{\varepsilon}\right) g_{\varepsilon}\left(k u_{\varepsilon}-w\right)-f(u) g(k u-w)\right] e_{\varepsilon}^{2 n+1} .
\end{aligned}
$$


Fix $x \in \Omega$, and consider the two intervals

$$
I_{1}:=\left[0, \varepsilon^{\frac{1}{1-p}}\right] \quad \text { and } \quad I_{2}:=\left[k^{-1} w(x), k^{-1}\left(w(x)+\varepsilon^{\frac{1}{1-q}}\right)\right] .
$$

There are three possibilities:-

(i) At least one of $u(x)$ and $u_{\varepsilon}(x)$ is in neither interval;

(ii) $\quad u(x)$ and $u_{\varepsilon}(x)$ are in different intervals;

(iii) $u(x)$ and $u_{\varepsilon}(x)$ are in the same interval.

For case (i), if $u(x) \in\left(\mathbf{R} \backslash I_{1}\right) \cap\left(\mathbf{R} \backslash I_{2}\right)$, then

$$
\left[f_{\varepsilon}\left(u_{\varepsilon}(x)\right) g_{\varepsilon}\left(k u_{\varepsilon}(x)-w(x)\right)-f_{\varepsilon}(u(x)) g_{\varepsilon}(k u(x)-w(x))\right] e_{\varepsilon}(x) \leq 0
$$

by the monotonicity of $f_{\varepsilon}$ and $g_{\varepsilon}$, and so the integrand on the RHS of (3.9) is nonpositive. If $u_{\varepsilon}(x) \in\left(\mathbf{R} \backslash I_{1}\right) \cap\left(\mathbf{R} \backslash I_{2}\right), f$ and $g$ replace $f_{\varepsilon}$ and $g_{\varepsilon}$ and the same result holds.

For case (ii), suppose that $u(x) \in I_{1} \backslash I_{2}$ and $u_{\varepsilon}(x) \in I_{2} \backslash I_{1}$. Then $u(x) \notin I_{2} \Rightarrow$ $g(k u(x)-w(x))=g_{\varepsilon}(k u(x)-w(x))$, and $u_{\varepsilon}(x) \notin I_{1} \Rightarrow f_{\varepsilon}\left(u_{\varepsilon}(x)\right)=f\left(u_{\varepsilon}(x)\right)$, and so, as

$$
\begin{aligned}
& g_{\varepsilon}\left(k u_{\varepsilon}(x)-w(x)\right)\left[f\left(u_{\varepsilon}(x)\right)-f(u(x))\right] e_{\varepsilon}(x) \\
& \quad+f(u(x))\left[g_{\varepsilon}\left(k u_{\varepsilon}(x)-w(x)\right)-g_{\varepsilon}(k u(x)-w(x))\right] e_{\varepsilon}(x) \leq 0
\end{aligned}
$$

by the monotonicity of $f$ and $g_{\varepsilon}$, it follows that the integrand on the RHS of (3.9) is again non-positive. A similar argument holds if $u_{\varepsilon}(x) \in I_{1} \backslash I_{2}$ and $u(x) \in I_{2} \backslash I_{1}$.

Hence the only positive contribution to the integrand of the RHS of (3.9) comes from case (iii), when $e_{\varepsilon}(x) \leq C \varepsilon^{\frac{1}{1-p}}=\max \left\{\varepsilon^{\frac{1}{1-p}}, k^{-1} \varepsilon^{\frac{1}{1-q}}\right\}$ for $p \leq q$. Hence from $(3.9)$,

$$
\left\|e_{\varepsilon}\right\|_{0,2(n+1), \Omega} \leq\left(C \frac{(n+1)^{2}}{2 n+1}\right)^{\frac{1}{2(n+1)}}\left(C \varepsilon^{\frac{1}{1-p}}\right)^{\frac{2 n+1}{2(n+1)}},
$$

and so in the limit as $n \rightarrow \infty,\left\|e_{\varepsilon}\right\|_{0, \infty, \Omega} \leq C \varepsilon^{\frac{1}{1-p}}$. Recalling that $k u_{\varepsilon}-v_{\varepsilon} \equiv k u-v$, it immediately follows that $\left\|v-v_{\varepsilon}\right\|_{0, \infty, \Omega} \leq C k \varepsilon^{\frac{1}{1-p}}$.

Similarly to (2.36), it is easy to establish the following result:

\section{Lemma 3.2}

$$
\left|u-u_{\varepsilon}\right|_{1, \Omega}+\left|v-v_{\varepsilon}\right|_{1, \Omega} \leq C\left[\underline{m}\left(\Omega_{\varepsilon}(u)\right)+\underline{m}\left(\Omega_{\varepsilon}(v)\right)\right]^{\frac{1}{2}} \varepsilon^{\frac{1+p}{2(1-p)}},
$$

where

$$
\Omega_{\varepsilon}(u):=\left\{x \in \Omega: 0<u(x) \leq \varepsilon^{\frac{1}{1-p}}\right\},
$$

and

$$
\Omega_{\varepsilon}(v):=\left\{x \in \Omega: 0<(k u-w)(x) \equiv v(x) \leq \varepsilon^{\frac{1}{1-q}}\right\} .
$$


Throughout the rest of this paper error bounds will be proved for the approximation of $u$. Clearly these same error bounds will also apply to $v$ and its corresponding approximation.

\subsection{Exact Integration}

In the notation of Section 2, the discretisation, with exact integration, of $\mathcal{Q}_{\varepsilon}$ leads to the following decoupled problem, the discrete, regularised analogue of the first problem of (3.2):

$$
\mathcal{Q}_{\varepsilon}^{h} \ldots\left\{\begin{aligned}
\text { Given the data of } \mathcal{Q}_{\varepsilon}, \text { find } u_{\varepsilon}^{h} \in S^{h} \text { such that } & \\
\left.u_{\varepsilon}^{h}\right|_{\partial \Omega^{h}} & =\left.\pi_{h} a\right|_{\partial \Omega^{h}}, \\
\int_{\Omega^{h}} \nabla u_{\varepsilon}^{h} \cdot \nabla \chi+\lambda \int_{\Omega^{h}} f_{\varepsilon}\left(u_{\varepsilon}^{h}\right) g_{\varepsilon}\left(k u_{\varepsilon}^{h}-w^{h}\right) \chi & =0 \quad \forall \chi \in S_{0}^{h},
\end{aligned}\right.
$$

where $w^{h}$, the solution of the discretisation of (3.1), satisfies $w^{h} \equiv Q^{h} w \in S^{h}$ by definition.

The existence and uniqueness of $u_{\varepsilon}^{h}$ follow as for $\mathcal{Q}$. By discrete Sobolev imbedding, (2.9), (2.12) and the SMP applied to $w$,

$$
\left\|w^{h}\right\|_{0, \infty, \Omega^{h}} \leq C\left[\left(\ln \frac{1}{h}\right)^{\frac{1}{2}}\left|\pi_{h} w-w^{h}\right|_{1, \Omega^{h}}+\left\|\pi_{h} w\right\|_{0, \infty, \Omega^{h}}\right] \leq C .
$$

Consider the auxiliary problem:

$$
\mathcal{Q}_{\varepsilon}^{*} \ldots\left\{\begin{aligned}
\text { Find } u_{\varepsilon}^{*} \text { such that } & \\
\left.u_{\varepsilon}^{*}\right|_{\partial \Omega} & =\left.a\right|_{\partial \Omega}, \\
-\Delta u_{\varepsilon}^{*}+\lambda f_{\varepsilon}\left(u_{\varepsilon}^{*}\right) g_{\varepsilon}\left(k u_{\varepsilon}^{*}-\bar{w}^{h}\right) & =0 \text { in } \Omega,
\end{aligned}\right.
$$

where

$$
\bar{w}^{h}(x):= \begin{cases}w^{h}(x) & x \in \bar{\Omega}^{h}, \\ w(x) & x \in \Omega \backslash \bar{\Omega}^{h} .\end{cases}
$$

Once again, existence, uniqueness and regularity follow as for $\mathcal{Q}$, and from the SMP,

$$
0 \leq u_{\varepsilon}^{*} \leq\|a\|_{0, \infty, \partial \Omega}
$$

Similarly to Lemma 3.1, it is easy to establish the following result:

\section{Lemma 3.3}

$$
\left\|u_{\varepsilon}-u_{\varepsilon}^{*}\right\|_{0, \infty, \Omega} \leq C\left\|\left(I-Q^{h}\right) w\right\|_{0, \infty, \Omega^{h}} \cdot \bullet
$$


Lemma 3.4 Under assumption B1, for h sufficiently small,

$$
\left|u_{\varepsilon}-u_{\varepsilon}^{h}\right|_{1, \Omega^{h}} \leq C h, \quad \text { and hence } \quad\left\|u_{\varepsilon}^{h}\right\|_{0, \infty, \Omega^{h}} \leq C .
$$

Proof: By (2.11), $\mathcal{Q}_{\varepsilon}^{*}, \mathcal{Q}_{\varepsilon}^{h}$, and the monotonicity of $f_{\varepsilon}$ and $g_{\varepsilon}$,

$$
\begin{aligned}
\left|Q^{h} u_{\varepsilon}^{*}-u_{\varepsilon}^{h}\right|_{1, \Omega^{h}}^{2} & =\lambda \int_{\Omega^{h}}\left[f_{\varepsilon}\left(u_{\varepsilon}^{h}\right) g_{\varepsilon}\left(k u_{\varepsilon}^{h}-w^{h}\right)-f_{\varepsilon}\left(u_{\varepsilon}^{*}\right) g_{\varepsilon}\left(k u_{\varepsilon}^{*}-w^{h}\right)\right]\left(Q^{h} u_{\varepsilon}^{*}-u_{\varepsilon}^{h}\right) \\
& \leq \lambda \int_{\Omega^{h}}\left[f_{\varepsilon}\left(u_{\varepsilon}^{h}\right) g_{\varepsilon}\left(k u_{\varepsilon}^{h}-w^{h}\right)-f_{\varepsilon}\left(u_{\varepsilon}^{*}\right) g_{\varepsilon}\left(k u_{\varepsilon}^{*}-w^{h}\right)\right]\left(Q^{h}-I\right) u_{\varepsilon}^{*} .
\end{aligned}
$$

For $u_{\varepsilon}^{h}<\varepsilon_{0}$, by the monotonicity of $f_{\varepsilon}, f_{\varepsilon}\left(u_{\varepsilon}^{h}\right)<f_{\varepsilon}\left(\varepsilon_{0}\right)=f\left(\varepsilon_{0}\right) \leq C$. For $u_{\varepsilon}^{h} \geq \varepsilon_{0}$ and the fact that, by requirement (2.26), $f^{\prime}$ is monotonically decreasing, $f_{\varepsilon}\left(u_{\varepsilon}^{h}\right)=f\left(u_{\varepsilon}^{h}\right) \leq f^{\prime}\left(\varepsilon_{0}\right)\left(u_{\varepsilon}^{h}-\varepsilon_{0}\right)+f\left(\varepsilon_{0}\right)$. Hence from (2.6), (2.9), (2.12) and (3.11),

$$
\begin{aligned}
\left\|f_{\varepsilon}\left(u_{\varepsilon}^{h}\right)\right\|_{0, \infty, \Omega^{h}} & \leq C\left(\left\|u_{\varepsilon}^{h}\right\|_{0, \infty, \Omega^{h}}+1\right) \\
& \leq C\left[\left(\ln \frac{1}{h}\right)^{\frac{1}{2}}\left|Q^{h} u_{\varepsilon}^{*}-u_{\varepsilon}^{h}\right|_{1, \Omega^{h}}+1\right] .
\end{aligned}
$$

An identical result follows for $\left\|g_{\varepsilon}\left(k u_{\varepsilon}^{h}-w^{h}\right)\right\|_{0, \infty, \Omega^{h}}$, by noting (3.10). Hence

$$
\left\|f_{\varepsilon}\left(u_{\varepsilon}^{h}\right)\right\|_{0, \infty, \Omega^{h}}\left\|g_{\varepsilon}\left(k u_{\varepsilon}^{h}-w^{h}\right)\right\|_{0, \infty, \Omega^{h}} \leq C\left[\left(\ln \frac{1}{h}\right)\left|Q^{h} u_{\varepsilon}^{*}-u_{\varepsilon}^{h}\right|_{1, \Omega^{h}}^{2}+1\right] .
$$

Now by (3.13), (3.11), (3.10), (3.15) and (2.12),

$$
\left|Q^{h} u_{\varepsilon}^{*}-u_{\varepsilon}^{h}\right|_{1, \Omega^{h}}^{2} \leq C h^{2}\left[\left(\ln \frac{1}{h}\right)\left|Q^{h} u_{\varepsilon}^{*}-u_{\varepsilon}^{h}\right|_{1, \Omega^{h}}^{2}+1\right]\left|u_{\varepsilon}^{*}\right|_{2, \Omega},
$$

and hence for $h$ sufficiently small,

$$
\left|Q^{h} u_{\varepsilon}^{*}-u_{\varepsilon}^{h}\right|_{1, \Omega^{h}} \leq C h .
$$

Similarly to Lemma 3.3 and noting (2.12), it follows readily that

$$
\left|u_{\varepsilon}-u_{\varepsilon}^{*}\right|_{1, \Omega}^{2} \leq C\left\|w-\bar{w}^{h}\right\|_{0,1, \Omega} \equiv C\left\|\left(I-Q^{h}\right) w\right\|_{0,1, \Omega^{h}} \leq C h^{2} .
$$

Hence the desired error bound in (3.12) follows from (3.17), (2.12) and (3.16). Finally combining this error bound with (2.6), (3.7), and (2.9), yields $\left\|u_{\varepsilon}^{h}\right\|_{0, \infty, \Omega^{h}} \leq$ $\left\|u_{\varepsilon}\right\|_{0, \infty, \Omega^{h}}+C h(\ln 1 / h)^{\frac{1}{2}} \leq C$, as required.

It now follows from (3.14), the analogous result for $g_{\varepsilon}\left(k u_{\varepsilon}^{h}-w^{h}\right)$ and Lemma 3.4 that under assumption B1,

$$
\left.\begin{array}{r}
\left\|f_{\varepsilon}\left(u_{\varepsilon}^{h}\right)\right\|_{0, \infty, \Omega^{h}} \\
\left\|g_{\varepsilon}\left(k u_{\varepsilon}^{h}-w^{h}\right)\right\|_{0, \infty, \Omega^{h}}
\end{array}\right\} \leq C .
$$


A near-optimal bound is now proved for the error $u_{\varepsilon}^{*}-u_{\varepsilon}^{h}$ by adapting the technique used by Nochetto in [15]. This involves introducing the following dual problem: Assuming $\mathrm{B} 1$, find $D \in H_{0}^{1}(\Omega) \cap H^{2}(\Omega)$ such that

$$
-\Delta D+\theta D=\delta
$$

where

$\theta(x):=\left\{\begin{array}{cl}\lambda\left(\frac{f_{\varepsilon}\left(u_{\varepsilon}^{*}\right) g_{\varepsilon}\left(k u_{\varepsilon}^{*}-w^{h}\right)-f_{\varepsilon}\left(u_{\varepsilon}^{h}\right) g_{\varepsilon}\left(k u_{\varepsilon}^{h}-w^{h}\right)}{u_{\varepsilon}^{*}-u_{\varepsilon}^{h}}\right)(x), & x \in \Omega^{h} \text { and } \\ 0, & \left(u_{\varepsilon}^{*}-u_{\varepsilon}^{h}\right)(x) \neq 0 \\ \text { otherwise },\end{array}\right.$

and $\delta$ is defined to be a regularisation of the Dirac delta function, satisfying

$$
0 \leq \delta \leq C(\gamma h)^{-2}, \quad \int_{\Omega} \delta=1
$$

and zero outside some open ball of radius $\gamma h$ about some point $x_{0}$, where $\gamma$ and $x_{0}$ are to be chosen.

Note that by the monotonicity of $f_{\varepsilon}$ and $g_{\varepsilon}$, the Mean Value Theorem, (3.5), (3.18) and (3.11),

$$
0 \leq \theta(x) \leq C \varepsilon^{-1} \quad \forall x \in \bar{\Omega},
$$

and hence there exists a unique solution to (3.19).

Lemma 3.5 Under assumption B1, the unique solution D of (3.19) satisfies

$$
\|\Delta D\|_{0,1, \Omega}+\|\theta D\|_{0,1, \Omega} \leq C
$$

Proof: See Lemma 2.1 in [15].

Lemma 3.6 Under assumptions BD, for h sufficiently small,

$$
\left\|\left(I-Q^{h}\right) D\right\|_{0,1, \Omega} \leq C\left(h \ln \frac{1}{h}\right)^{2}
$$

Proof: See Lemma 2.2 in [15].

Lemma 3.7 Under assumptions BD, there exists $h_{0}$ such that $\forall h<h_{0}$,

$$
\left\|u_{\varepsilon}^{*}-u_{\varepsilon}^{h}\right\|_{0, \infty, \Omega^{h}} \leq C\left\|\left(I-Q^{h}\right) u_{\varepsilon}^{*}\right\|_{0, \infty, \Omega^{h}}+C\left(h \ln \frac{1}{h}\right)^{2} \text {. }
$$

Proof: Define $e^{h}:=Q^{h} u_{\varepsilon}^{*}-u_{\varepsilon}^{h} \in S_{0}^{h}$. Let $x_{0} \in \Omega^{h}$ be such that $\left\|e^{h}\right\|_{0, \infty, \Omega}=\left|e^{h}\left(x_{0}\right)\right|$, and, noting (2.7) and (3.20), choose $x_{1} \in B_{\gamma h}\left(x_{0}\right)$ satisfying

$$
e^{h}\left(x_{1}\right)=\int_{\Omega} e^{h} \delta=\int_{\Omega^{h}} e^{h} \delta
$$


By the Mean Value Theorem and (2.5),

$$
\left|e^{h}\left(x_{0}\right)\right| \leq\left|e^{h}\left(x_{1}\right)\right|+\gamma h\left\|e^{h}\right\|_{1, \infty, \Omega} \leq\left|e^{h}\left(x_{1}\right)\right|+C \gamma\left|e^{h}\left(x_{0}\right)\right|,
$$

and so for $\gamma$ sufficiently small,

$$
\left\|e^{h}\right\|_{0, \infty, \Omega}=\left|e^{h}\left(x_{0}\right)\right| \leq C\left|e^{h}\left(x_{1}\right)\right|=C\left|\int_{\Omega^{h}} e^{h} \delta\right|
$$

Note from $\mathcal{Q}_{\varepsilon}^{*}$ and $\mathcal{Q}_{\varepsilon}^{h}$ that for all $\chi \in S_{0}^{h}$,

$$
\int_{\Omega^{h}}\left[\nabla e^{h} \cdot \nabla \chi+\theta e \chi\right]=0
$$

where $e:=u_{\varepsilon}^{*}-u_{\varepsilon}^{h}\left(=e^{h}+\left(I-Q^{h}\right) u_{\varepsilon}^{*}\right)$. As $e^{h} \in S_{0}^{h}$, by (3.19), and (3.23),

$$
\begin{aligned}
\int_{\Omega^{h}} e^{h} \delta & =\int_{\Omega^{h}}\left[\nabla e^{h} \cdot \nabla D+\theta e^{h} D\right] \\
& =\int_{\Omega^{h}}\left[\nabla e^{h} \cdot \nabla\left(I-Q^{h}\right) D+\theta\left(e^{h} D-e Q^{h} D\right)\right] \\
& =\int_{\Omega^{h}}\left[\theta e\left(I-Q^{h}\right) D-\theta D\left(I-Q^{h}\right) u_{\varepsilon}^{*}\right] .
\end{aligned}
$$

Hence the desired result follows from (3.22), (3.24), the definition of $\theta,(3.10),(3.11)$, (3.18), Lemma 3.6 and Lemma 3.5 .

All that is now necessary to prove a bound on $\left\|u-u_{\varepsilon}^{h}\right\|_{0, \infty, \Omega}$ is a bound on $\left\|\left(I-Q^{h}\right) u_{\varepsilon}^{*}\right\|_{0, \infty, \Omega^{h}}$ and $\left\|\left(I-Q^{h}\right) w\right\|_{0, \infty, \Omega^{h}}$; that is, the generalisation of (2.13) to more general boundary data. The above analysis is readily modified to prove such a bound.

Lemma 3.8 If $\psi \in W^{2, \infty}(\Omega)$ then, under assumptions BD, there exists $h_{0}>0$ such that for all $h<h_{0}$,

$$
\left\|\left(I-Q^{h}\right) \psi\right\|_{0, \infty, \Omega^{h}} \leq C\left(h \ln \frac{1}{h}\right)^{2}
$$

Proof: Consider the dual problem (3.19) with $\theta \equiv 0$ : clearly Lemmas 3.5 and 3.6 still hold. In addition, by (3.20), and the Calderón-Zygmund inequality (2.1), $D \equiv \mathcal{G} \delta$ satisfies for all $\alpha \in(0,1)$

$$
|\mathcal{G} \delta|_{2,1, \Omega} \leq C|\mathcal{G} \delta|_{2,1+\alpha, \Omega} \leq C \alpha^{-1}\|\delta\|_{0,1+\alpha, \Omega} \leq C \alpha^{-1}(\gamma h)^{-2 \alpha^{\prime}}
$$

where $\alpha^{\prime}:=\alpha /(1+\alpha)$. Choosing $\alpha:=C(\ln 1 / h)^{-1}$, yields that

$$
|D|_{2,1, \Omega} \leq C\left(\ln \frac{1}{h}\right)
$$


Adapting the argument of Lemma 3.7 with $e^{h}:=\left(Q^{h}-\pi_{h}\right) \psi$ and using a trace inequality yields that

$$
\begin{aligned}
\left\|\left(Q^{h}-\pi_{h}\right) \psi\right\|_{0, \infty, \Omega} \leq & C\left|\int_{\Omega^{h}}\left(Q^{h}-\pi_{h}\right) \psi \delta\right|=C\left|\int_{\Omega^{h}} \nabla\left(Q^{h}-\pi_{h}\right) \psi \cdot \nabla D\right| \\
& =C\left|\int_{\Omega^{h}} \nabla\left(I-\pi_{h}\right) \psi \cdot \nabla\left[D+\left(Q^{h}-I\right) D\right]\right| \\
\leq & C||\left(I-\pi_{h}\right) \psi\left\|_{0, \infty, \Omega}|| D\right\|_{2,1, \Omega} \\
& \quad+C\left|\left(I-\pi_{h}\right) \psi\right|_{1, \infty, \Omega}\left|\left(I-Q^{h}\right) D\right|_{1,1, \Omega}
\end{aligned}
$$

The desired result then follows from (3.26), (2.5), (2.9) and Lemma 3.6.

Theorem 3.1 There exists $h_{0}>0$ such that $\forall h<h_{0}$ and for $\varepsilon=C(h \ln 1 / h)^{2(1-p)}$ :

(a) Under boundary assumption B1,

$$
\left|u-u_{\varepsilon}^{h}\right|_{1, \Omega^{h}} \leq C h ;
$$

(b) Under boundary assumption BD,

$$
\left\|u-u_{\varepsilon}^{h}\right\|_{0, \infty, \Omega^{h}} \leq C\left(h \ln \frac{1}{h}\right)^{2} .
$$

Proof: The $H^{1}$ bound follows from Lemma 3.2 and Lemma 3.4.

Note the splittings $\left(I-Q^{h}\right) u_{\varepsilon}^{*} \equiv\left(I-Q^{h}\right) a+\left(I-Q^{h}\right) \mathcal{G}\left[\Delta a-\lambda f_{\varepsilon}\left(u_{\varepsilon}^{*}\right) g_{\varepsilon}\left(k u_{\varepsilon}^{*}-\bar{w}^{h}\right)\right]$, and $\left(I-Q^{h}\right) w \equiv\left(I-Q^{h}\right) c+\left(I-Q^{h}\right) \mathcal{G} \Delta c$, where $a, c \in W^{2, \infty}(\Omega)$. It now follows from (2.14), (3.11), and Lemma 3.8 that

$$
\left\|\left(I-Q^{h}\right) u_{\varepsilon}^{*}\right\|_{0, \infty, \Omega^{h}}+\left\|\left(I-Q^{h}\right) w\right\|_{0, \infty, \Omega^{h}} \leq C\left(h \ln \frac{1}{h}\right)^{2} .
$$

The $L^{\infty}$ result is now an immediate consequence of Lemma 3.1, Lemma 3.3, (3.28) and Lemma 3.7.

It now follows from Theorem 3.1 that under the appropriate non-degeneracy conditions, results analogous to (2.18) and (2.19) hold.

Note that the techniques of [6] can now be used to prove that optimal $H^{1}$ and $L^{\infty}$ error bounds apply if $\mathcal{Q}$ is approximated directly, using a non-regularised scheme with exact integration. However, in the light of the results of Section 2 for the catalyst problem, the more practical scheme involving quadrature, analysed in the following section, is applied to the regularised problem. 


\subsection{Numerical Integration}

Using the quadrature rule introduced in Section 2, the discrete problem with numerical integration corresponding to $\mathcal{Q}_{\varepsilon}^{h}$ is stated as follows:

$$
\hat{\mathcal{Q}}_{\varepsilon}^{h} \ldots\left\{\begin{aligned}
\text { Given the data of } \mathcal{Q} \varepsilon & \text { find } \hat{u}_{\varepsilon}^{h} \in S^{h} \text { such that } \\
\left.\hat{u}_{\varepsilon}^{h}\right|_{\partial \Omega^{h}} & =\left.\pi_{h} a\right|_{\partial \Omega^{h}}, \\
\int_{\Omega^{h}} \nabla \hat{u}_{\varepsilon}^{h} \cdot \nabla \chi+\lambda\left(f_{\varepsilon}\left(\hat{u}_{\varepsilon}^{h}\right) g_{\varepsilon}\left(\hat{u}_{\varepsilon}^{h}-w^{h}\right), \chi\right)^{h} & =0 \forall \chi \in S_{0}^{h} .
\end{aligned}\right.
$$

The existence and uniqueness of a solution of $\hat{\mathcal{Q}}_{\varepsilon}^{h}$ are readily established as above.

Lemma 3.9 Under assumptions B1, for $h<h_{0}$,

$$
\left|\hat{u}_{\varepsilon}^{h}-Q^{h} u_{\varepsilon}\right|_{1, \Omega^{h}} \leq C \varepsilon^{-1} h^{2}\left(\ln \frac{1}{h}\right)^{\frac{1}{2}} .
$$

Proof: Define $\hat{E}:=\hat{u}_{\varepsilon}^{h}-Q^{h} u_{\varepsilon} \in S_{0}^{h}$. By (2.11), $\mathcal{Q}_{\varepsilon}, \hat{\mathcal{Q}}_{\varepsilon}^{h}$ and (2.22),

$$
\begin{aligned}
|\hat{E}|_{1, \Omega^{h}}^{2}=\lambda \int_{\Omega^{h}} & f_{\varepsilon}\left(u_{\varepsilon}\right) g_{\varepsilon}\left(k u_{\varepsilon}-w\right) \hat{E}-\lambda\left(f_{\varepsilon}\left(\hat{u}_{\varepsilon}^{h}\right) g_{\varepsilon}\left(\hat{u}_{\varepsilon}^{h}-w^{h}\right), \hat{E}\right)^{h} \\
\leq C h^{2}|\hat{E}|_{1, \Omega^{h}} & {\left[\left(\ln \frac{1}{h}\right)^{\frac{1}{2}}\left|f_{\varepsilon}\left(u_{\varepsilon}\right) g_{\varepsilon}\left(k u_{\varepsilon}-w\right)\right|_{2,1, \Omega^{h}}+\left|\pi_{h}\left[f_{\varepsilon}\left(u_{\varepsilon}\right) g_{\varepsilon}\left(k u_{\varepsilon}-w\right)\right]\right|_{1, \Omega^{h}}\right] } \\
& +\lambda\left(f_{\varepsilon}\left(u_{\varepsilon}\right) g_{\varepsilon}\left(k u_{\varepsilon}-w\right)-f_{\varepsilon}\left(\hat{u}_{\varepsilon}^{h}\right) g_{\varepsilon}\left(k \hat{u}_{\varepsilon}^{h}-w^{h}\right), \hat{E}\right)^{h}
\end{aligned}
$$

noting that $k u_{\varepsilon}-w=v_{\varepsilon} \geq 0$ by (3.7).

By (3.7), the Mean Value Theorem, (3.5), (2.23), the monotonicity of $f_{\varepsilon}$ and $g_{\varepsilon}$ and Poincaré's inequality,

$$
\begin{aligned}
& \left(f_{\varepsilon}\left(u_{\varepsilon}\right) g_{\varepsilon}\left(k u_{\varepsilon}-w\right)-f_{\varepsilon}\left(\hat{u}_{\varepsilon}^{h}\right) g_{\varepsilon}\left(k \hat{u}_{\varepsilon}^{h}-w^{h}\right), \hat{E}\right)^{h}= \\
& \left(f_{\varepsilon}\left(u_{\varepsilon}\right)\left[g_{\varepsilon}\left(k u_{\varepsilon}-w\right)-g_{\varepsilon}\left(k u_{\varepsilon}-w^{h}\right)\right], \hat{E}\right)^{h} \\
& \quad+\left(f_{\varepsilon}\left(u_{\varepsilon}\right) g_{\varepsilon}\left(k u_{\varepsilon}-w^{h}\right)-f_{\varepsilon}\left(\hat{u}_{\varepsilon}^{h}\right) g_{\varepsilon}\left(k \hat{u}_{\varepsilon}^{h}-w^{h}\right),\left(\hat{u}_{\varepsilon}^{h}-u_{\varepsilon}\right)+\left(I-Q^{h}\right) u_{\varepsilon}\right)^{h} \\
& \leq C \varepsilon^{-1} \| \pi_{h} w-\left.w^{h}\right|_{0, \Omega^{h}}|\hat{E}|_{1, \Omega^{h}} \\
& \quad+\left(f_{\varepsilon}\left(u_{\varepsilon}\right) g_{\varepsilon}\left(k u_{\varepsilon}-w^{h}\right)-f_{\varepsilon}\left(\hat{u}_{\varepsilon}^{h}\right) g_{\varepsilon}\left(k \hat{u}_{\varepsilon}^{h}-w^{h}\right),\left(I-Q^{h}\right) u_{\varepsilon}\right)^{h} .
\end{aligned}
$$

Note that exactly as in Lemma 3.4, with $\hat{u}_{\varepsilon}^{h}$ replacing $u_{\varepsilon}^{h}$ in (3.14),

$$
\left\|f_{\varepsilon}\left(\hat{u}_{\varepsilon}^{h}\right)\right\|_{0, \infty, \Omega^{h}} \leq C\left(\left\|\hat{u}_{\varepsilon}^{h}\right\|_{0, \infty, \Omega^{h}}+1\right) .
$$


Hence by the Mean Value Theorem, (3.5), (3.7), (2.23), (3.31), discrete Sobolev imbedding (2.6) and Poincaré's inequality,

$$
\begin{aligned}
& \left(f_{\varepsilon}\left(u_{\varepsilon}\right) g_{\varepsilon}\left(k u_{\varepsilon}-w^{h}\right)-f_{\varepsilon}\left(\hat{u}_{\varepsilon}^{h}\right) g_{\varepsilon}\left(k \hat{u}_{\varepsilon}^{h}-w^{h}\right),\left(I-Q^{h}\right) u_{\varepsilon}\right)^{h} \\
& \leq C \varepsilon^{-1}\left(1+\left\|\hat{u}_{\varepsilon}^{h}\right\|_{0, \infty, \Omega^{h}}\right)\left\|\pi_{h} u_{\varepsilon}-\hat{u}_{\varepsilon}^{h}\right\|_{0, \Omega^{h}}\left\|\left(\pi_{h}-Q^{h}\right) u_{\varepsilon}\right\|_{0, \Omega^{h}} \\
& \leq C \varepsilon^{-1}\left[1+\left(\ln \frac{1}{h}\right)^{\frac{1}{2}}\left(\left|\hat{u}_{\varepsilon}^{h}-\pi_{h} u_{\varepsilon}\right|_{1, \Omega^{h}}\right)\right]\left\|\pi_{h} u_{\varepsilon}-\hat{u}_{\varepsilon}^{h}\right\|_{0, \Omega^{h}}\left\|\left(\pi_{h}-Q^{h}\right) u_{\varepsilon}\right\|_{0, \Omega^{h}} \\
& \leq C \varepsilon^{-1}\left(\left\|\left(\pi_{h}-Q^{h}\right) u_{\varepsilon}\right\|_{0, \Omega^{h}}^{2}+\left\|\left(\pi_{h}-Q^{h}\right) u_{\varepsilon}\right\|_{0, \Omega^{h}}|\hat{E}|_{1, \Omega^{h}}\right) \\
& \quad+C \varepsilon^{-1}\left(\ln \frac{1}{h}\right)^{\frac{1}{2}}\left(|\hat{E}|_{1, \Omega^{h}}^{2}+\left|\left(\pi_{h}-Q^{h}\right) u_{\varepsilon}\right|_{1, \Omega^{h}}^{2}\right)\left\|\left(\pi_{h}-Q^{h}\right) u_{\varepsilon}\right\|_{0, \Omega^{h}} .
\end{aligned}
$$

Hence by (3.30), (3.32), (2.9) and (2.12),

$$
\begin{aligned}
& \left(f_{\varepsilon}\left(u_{\varepsilon}\right) g_{\varepsilon}\left(k u_{\varepsilon}-w\right)-f_{\varepsilon}\left(\hat{u}_{\varepsilon}^{h}\right) g_{\varepsilon}\left(k \hat{u}_{\varepsilon}^{h}-w^{h}\right), \hat{E}\right)^{h} \leq \\
& C \varepsilon^{-1} h^{2}|\hat{E}|_{1, \Omega}+C \varepsilon^{-1} h^{2}\left(\ln \frac{1}{h}\right)^{\frac{1}{2}}|\hat{E}|_{1, \Omega}^{2}+C \varepsilon^{-1} h^{4}\left(\ln \frac{1}{h}\right)^{\frac{1}{2}} .
\end{aligned}
$$

By expansion, (3.5) and the fact that $\forall t \geq 0, f_{\varepsilon}^{\prime}(t), g_{\varepsilon}^{\prime}(t) \geq 0$, and for almost all $t \geq 0, f_{\varepsilon}^{\prime \prime}(t), g_{\varepsilon}^{\prime \prime}(t) \leq 0$,

$$
\begin{gathered}
\left|f_{\varepsilon}\left(u_{\varepsilon}\right) g_{\varepsilon}\left(k u_{\varepsilon}-w\right)\right|_{2,1, \Omega^{h}} \leq-C \int_{\Omega}\left[f_{\varepsilon}^{\prime \prime}\left(u_{\varepsilon}\right)\left(\nabla u_{\varepsilon}\right)^{2}+g_{\varepsilon}^{\prime \prime}\left(k u_{\varepsilon}-w\right)\left(\nabla\left(k u_{\varepsilon}-w\right)\right)^{2}\right] \\
\quad+\int_{\Omega}\left[\left(\nabla u_{\varepsilon}\right)^{2}+\left(\nabla\left(k u_{\varepsilon}-w\right)\right)^{2}\right] f_{\varepsilon}^{\prime}\left(u_{\varepsilon}\right) g_{\varepsilon}^{\prime}\left(k u_{\varepsilon}-w\right)+C \varepsilon^{-1}\left|u_{\varepsilon}\right|_{2,1, \Omega} .
\end{gathered}
$$

From exactly the same approach used to derive (2.33), it follows that

$$
\left.\begin{array}{c}
-\int_{\Omega} f_{\varepsilon}^{\prime \prime}\left(u_{\varepsilon}\right)\left(\nabla u_{\varepsilon}\right)^{2} \\
-\int_{\Omega} g_{\varepsilon}^{\prime \prime}\left(k u_{\varepsilon}-w\right)\left(\nabla\left(k u_{\varepsilon}-w\right)\right)^{2}
\end{array}\right\} \leq C \varepsilon^{-1}\left\|u_{\varepsilon}\right\|_{2,1, \Omega} .
$$

By (3.5) and a trace inequality,

$$
\begin{aligned}
\int_{\Omega}\left(\nabla u_{\varepsilon}\right)^{2} f_{\varepsilon}^{\prime}\left(u_{\varepsilon}\right) g_{\varepsilon}^{\prime}\left(k u_{\varepsilon}-w\right) & =\int_{\Omega} g_{\varepsilon}^{\prime}\left(k u_{\varepsilon}-w\right) \nabla u_{\varepsilon} \cdot \nabla f_{\varepsilon}\left(u_{\varepsilon}\right) \\
& \leq C \varepsilon^{-1} \int_{\Omega} \nabla u_{\varepsilon} \cdot \nabla f_{\varepsilon}\left(u_{\varepsilon}\right)
\end{aligned}
$$




$$
\begin{aligned}
& =C \varepsilon^{-1}\left(\int_{\partial \Omega} f_{\varepsilon}\left(u_{\varepsilon}\right) \nabla u_{\varepsilon} \cdot \underline{\mathbf{n}}-\int_{\Omega} f_{\varepsilon}\left(u_{\varepsilon}\right) \Delta u_{\varepsilon}\right) \\
& \leq C \varepsilon^{-1}\left\|u_{\varepsilon}\right\|_{2,1, \Omega} .
\end{aligned}
$$

Similarly

$$
\begin{gathered}
\int_{\Omega}\left(\nabla\left(k u_{\varepsilon}-w\right)\right)^{2} f_{\varepsilon}^{\prime}\left(u_{\varepsilon}\right) g_{\varepsilon}^{\prime}\left(k u_{\varepsilon}-w\right) \leq C \varepsilon^{-1} \int_{\Omega} \nabla\left(k u_{\varepsilon}-w\right) \cdot \nabla g_{\varepsilon}\left(k u_{\varepsilon}-w\right) \\
\leq C \varepsilon^{-1}\left(\left\|u_{\varepsilon}\right\|_{2,1, \Omega}+\|w\|_{2,1, \Omega}\right) .
\end{gathered}
$$

Hence, by (3.34), (3.35), (3.36) and (3.37),

$$
\left|f_{\varepsilon}\left(u_{\varepsilon}\right) g_{\varepsilon}\left(k u_{\varepsilon}-w\right)\right|_{2,1, \Omega^{h}} \leq C \varepsilon^{-1} .
$$

Setting $\phi_{\varepsilon}:=f_{\varepsilon}\left(u_{\varepsilon}\right) g_{\varepsilon}\left(k u_{\varepsilon}-w\right)$, it now follows from (2.9), Sobolev imbedding, as $W^{2,1}\left(\Omega^{h}\right) \subseteq W^{1,2}\left(\Omega^{h}\right),(3.38)$ and $(3.5)$ that

$$
\begin{aligned}
\left|\pi_{h} \phi_{\varepsilon}\right|_{1, \Omega^{h}} & \leq\left|\left(\pi_{h}-I\right) \phi_{\varepsilon}\right|_{1, \Omega^{h}}+\left|\phi_{\varepsilon}\right|_{1, \Omega^{h}} \leq C\left\|\phi_{\varepsilon}\right\|_{2,1, \Omega^{h}} \\
& \leq C\left(\left|\phi_{\varepsilon}\right|_{2,1, \Omega^{h}}+\varepsilon^{-1}\right) \leq C \varepsilon^{-1} .
\end{aligned}
$$

By (3.29), (3.38), (3.39) and (3.33), provided that $\varepsilon$ is such that

$$
\varepsilon^{-1} h^{2}\left(\ln \frac{1}{h}\right)^{\frac{1}{2}} \rightarrow 0
$$

as $h \rightarrow 0$, then for $h$ sufficiently small,

$$
|\hat{E}|_{1, \Omega^{h}}^{2} \leq C \varepsilon^{-1} h^{2}\left[\left(\ln \frac{1}{h}\right)^{\frac{1}{2}}+1\right]|\hat{E}|_{1, \Omega^{h}}+C \varepsilon^{-1} h^{4}\left(\ln \frac{1}{h}\right)^{\frac{1}{2}},
$$

and the required result now follows.

Theorem 3.2 There exists $h_{0}>0$ such that for all $h<h_{0}$, and for $\varepsilon=\left(h^{2} \ln 1 / h\right)^{\frac{1-p}{2-p}}$ :

(a) Under boundary assumption B1,

$$
\left|u-\hat{u}_{\varepsilon}^{h}\right|_{1, \Omega^{h}} \leq \begin{cases}C h, & p>\frac{1}{2}, \\ C\left(h^{2} \ln \frac{1}{h}\right)^{\frac{1+p}{2(2-p)}} & , 0<p \leq \frac{1}{2} ;\end{cases}
$$

(b) Under boundary assumption BQ,

$$
\left\|u-\hat{u}_{\varepsilon}^{h}\right\|_{0, \infty, \Omega^{h}} \leq C\left(h^{2} \ln \frac{1}{h}\right)^{\frac{1}{2-p}} .
$$


Proof: Without making any assumptions about non-degeneracy, the $H^{1}$ bound follows from Lemma 3.2, (2.12) and Lemma 3.9.

Lemma 3.1, discrete Sobolev imbedding (2.6), Lemma 3.9 and noting that (3.28) holds with $u_{\varepsilon}^{*}$ replaced by $u_{\varepsilon}$, yield that

$$
\left\|u-\hat{u}_{\varepsilon}^{h}\right\|_{0, \infty, \Omega^{h}} \leq C \varepsilon^{\frac{1}{1-p}}+C\left(h \ln \frac{1}{h}\right)^{2}+C \varepsilon^{-1} h^{2} \ln \frac{1}{h} .
$$

The required $L^{\infty}$ bound now follows by setting $\varepsilon=\left(h^{2} \ln 1 / h\right)^{\frac{1-p}{2-p}}$.

Note that the $H^{1}$ bound obtained above can be improved under an assumption of non-degeneracy. Suppose that under certain boundary assumptions it can be shown that $\underline{m}\left(\Omega_{\varepsilon}(u)\right), \underline{m}\left(\Omega_{\varepsilon}(v)\right) \leq C \varepsilon^{\frac{1}{2}}$, as is the case for the catalyst problem in Section 2, with $f(t) \equiv[t]_{+}^{p}$. Then exactly as in Lemma 2.2, it follows that

$$
\left|u-\hat{u}_{\varepsilon}^{h}\right|_{1, \Omega^{h}} \leq \begin{cases}C h, & p>\frac{1}{3}, \\ C\left(h^{2} \ln \frac{1}{h}\right)^{\frac{3+p}{4(2-p)}} & , 0<p \leq \frac{1}{3} .\end{cases}
$$

Finally, it is noted that the above analysis can be easily modified to deal with the cases

$$
\text { (i) } p=q=1 ; \quad \text { (ii) } 0<p<q=1 \text {. }
$$

Case (i) requires no regularisation, and in case (ii), only $f$ need be regularised.

The easiest adaptation of the above methods would involve the truncation of $g$, (and/or $f$, as appropriate) in a manner similar to (2.10), to give globally Lipschitz functions. It is then not difficult to see that for case (ii), Theorems 3.1 and 3.2 hold, and that for case (i), Theorem 3.1 holds, and the $L^{\infty}$ bound of Theorem 3.2 is modified to $C(h \ln 1 / h)^{2}$.

\section{References}

[1] Adams, R.A., Sobolev Spaces. Academic Press, New York.

[2] Alt, H.W. \& Phillips, D., 1986, A free boundary problem for semilinear elliptic equations. J. Reine Angew. Math. 368, pp 63-107.

[3] Aris, R., 1975, The Mathematical Theory of Diffusion and Reaction in Permeable Catalysts. Clarendon Press, Oxford.

[4] Barrett, J.W. \& Elliott, C.M., 1987, Total flux estimates for a finite element approximation of elliptic equations. IMA J. of Num. Anal. 7, pp 129-148.

[5] Barrett, J.W. \& Knabner, P., 1992, Finite element approximation of the transport of reactive solutes in porous media, Part 1: Error estimates for nonequilibrium adsorption processes. Submitted for publication. 
[6] Barrett, J.W. \& Shanahan, R.M., 1991, Finite element approximation of a model reaction-diffusion problem with a non-Lipschitz nonlinearity. Numer. Math. 59, pp 217-242.

[7] Bobisud, L.E., \& Stakgold, I., 1987, Dead cores in nonlinear reaction-diffusion systems. Nonlinear Anal. 11, pp 1219-1227.

[8] Chan, C.Y., \& Fung, D.T., 1992, Dead cores and effectiveness of semilinear reaction-diffusion systems. J. Math. Anal. and Appl. 171, pp 498-515.

[9] Ciarlet, P.G., 1978, The Finite Element Method for Elliptic Problems. NorthHolland, Amsterdam.

[10] Crouzeix, M. \& Rappaz, J., 1990, On Numerical Approximation in Bifurcation Theory. Springer, Berlin.

[11] Friedman, A. \& Phillips, D., 1984, The free boundary of a semilinear elliptic equation. Trans. Am. Math. Soc. 282, pp 153-182.

[12] Gilbarg, D. \& Trudinger, N.S., 1983, Elliptic Partial Differential Equations of Second Order. 2nd Ed., Springer, Berlin.

[13] Kufner, A., John, O. \& Fučik, S., Function Spaces. Nordhoff, Leyden.

[14] Nochetto, R.H., 1986, A note on the approximation of free boundaries by finite elements. RAIRO Mod. Math. Anal. Numer. 20, pp 355-368.

[15] Nochetto, R.H., 1988, Sharp $L^{\infty}$ error estimates for semilinear elliptic problems with free boundaries. Numer. Math. 54, pp 243-255.

[16] Phillips, D., 1983, Hausdorff measure estimates of a free boundary for a minimum problem. Comm. P.D.E. 8, pp 1409-1454.

[17] Schatz, A.H., \& Wahlbin, L.B., 1982, On the quasi-optimality in $L^{\infty}$ of the

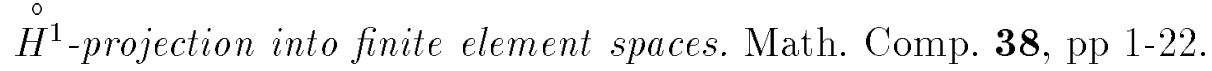

[18] Schultz, M.H., 1971, $L^{2}$ error bounds for the Rayleigh-Ritz-Galerkin method. SIAM J. Numer. Anal., 8, pp 737-748

[19] Stakgold, I., 1985, Reaction-diffusion problems in chemical engineering, in Nonlinear Diffusion Problems, edited by Fasano and Primicerio, Lecture Notes in Mathematics 1224, Springer-Verlag, Berlin.

[20] Stein, E.M., 1970, Singular Integrals and Differentiability Properties of Functions. Princeton Univ. Press, Princeton. 